\title{
Temps de travail des internes
}

Rédacteur en chef,

Inserm U.426 et

Département de physiologie, UFR de Médecine

Ce siècle avait deux ans!

Le 4 Ventose, an X - 23 février 1802, Bonaparte, encore premier consul, crée l'Internat des Hospices de Paris.

Pour la première fois en France, cette « école supérieure d'instruction pratique » permet de mettre en place un dispositif assurant la médicalisation de la continuité des soins dans les hôpitaux. Les internes, sélectionnés par concours, logent à l'hôpital et bénéficient de quatre ans de formation théorique et pratique.

1816. Les premières listes de garde sont affichées dans les bureaux de l'hôpital et dans les locaux réservés aux internes. Ceux-ci deviennent rapidement la cheville ouvrière du fonctionnement hospitalier.

1971. Mise en place du numerus clausus qui contingente le nombre de médecins formés chaque année. On connaît aujourd'hui les effets dévastateurs, en termes de pénurie médicale, de ces mesures pérennisées depuis trente ans et qui tardent encore à être amendées.

1984. Suppression des Certificats d'Études Spécialisées (CES), l'une des deux voies de formation des médecins spécialistes en France. Le concours de l'internat devient alors le passage obligé, la seule voie d'accession à une spécialité médicale.

Ce siècle avait deux ans...

Le 10 septembre 2002, le nouveau statut des internes est fixé par décret. Deux dispositions en modifient fondamentalement le cahier des charges: l'intégration des gardes dans les obligations hospitalières, d'une part, et l'obligation du repos de sécurité après chaque garde de nuit d'autre part. Les internes doivent désormais fournir onze demi-journées par semaine (la durée d'une «demijournée » n'est pas bien définie et les exégètes l'estiment comprise dans une fourchette de trois heures et demi à quatre heures et demi). Dans ces onze demi-journées sont désormais incluses les deux demi-journées qui équivalent à un lot composé d'une garde de nuit par semaine et d'une garde de dimanche par mois. Y sont également incluses deux demi-journées de formation universitaire.
II reste donc sept demijournées de travail «de jour » à l'hôpital auxquelles s'ajoutent, selon les services, quelques heures de «continuité de service » du samedi après-midi et du dimanche après-midi. Quant au repos de sécurité après une garde de nuit, repos qui revêt un caractère obligatoire en ce qui concerne l'exécution d'actes médicaux, il s'étend sur toute la journée qui suit la garde.

Pour faire court, on peut considérer que le temps de travail des internes, de 48 heures hebdomadaires au total, temps universitaire inclus, comprend une trentaine d'heures de travail dans les services hospitaliers.

Deux types de conséquences découlent de ces mesures, qui modifient en profondeur la formation des internes eux-mêmes et le fonctionnement des hôpitaux. La formation des internes a longtemps été conçue comme un compagnonnage. Le temps important qu'ils passaient à I'hôpital, leur association étroite aux pratiques de soins médicaux et chirurgicaux, les responsabilités qui leur étaient confiées dans ce contexte étaient des éléments forts d'une formation reconnue. La place de l'interne dans le dispositif hospitalier ne pourra bien sûr pas rester la même dans ce nouveau régime. La continuité des soins, si elle doit être assurée, amènera nécessairement à redéfinir les places, prérogatives et responsabilités de chacun.

L'aménagement et la réduction du temps de travail (ARTT) des médecins ne concerne pas que les internes. Les praticiens hospitaliers voient également leur activité réglementée. Dans un contexte évident de pénurie de médecins (et de personnel non médical, infirmiers et infirmières en tête...), que personne ne conteste, et de perspectives démographiques très sombres, c'est le fonctionnement même de nombreux services et centres hospitaliers qui est aujourd'hui menacé dans sa forme actuelle. C'est donc bien une remise en question globale 
de la prise en charge des malades que tous, soignants et soignés, sont invités à faire.

Cette situation est-elle une exception française? Quel est le statut des internes dans d'autres pays, en particulier en Europe et outre-Atlantique?

En Europe d'abord. L'harmonisation des horaires de travail entre les pays de la communauté a été décidée dès 1993. Elle entrera en vigueur le $1^{\text {er }}$ août 2004. Les heures de travail seront d'abord limitées à 58 heures hebdomadaires, puis 56 heures à compter d'août 2007 pour atteindre 48 heures par semaine en 2009 . On entend par temps de travail le temps passé à travailler sur le lieu de travail. Les mêmes directives imposent un repos continu de 11 heures par période de 24 heures et un temps de travail de 8 heures par 24 heures pour le travail de nuit [1]. Comme le souligne T. Pickersgill: « il nous faudra plus de médecins et une meilleure organisation pour satisfaire à la loi » [1].

Même préoccupation aux États-Unis. Un point très complet est fait dans le numéro du 17 octobre 2002 du New England Journal of Medicine [2-4]. Il y est indiqué que les cadences actuelles des residents sont infernales, 100 à 120 heures hebdomadaires. La fatigue est endémique et le manque de sommeil responsable de troubles de l'humeur. Quant à savoir si ce manque de sommeil est responsable d'une diminution des performances cliniques, le moins que l'on puisse dire est que les résultats des quelques études effectuées sont contradictoires, pour ne rien dire de la validité et de la pertinence des aspects méthodologiques de ces études. Néanmoins, des mesures de restriction des horaires de travail pour les résidents seront mises en place dès 2003. Un maximum de... 80 heures hebdomadaires est instauré, éventuellement augmenté de $10 \%$ en cas de force majeure! Transgresser ces règles exposerait les centres hospitalouniversitaires à une perte d'accréditation.

Cette réduction spectaculaire (!) des horaires de travail des résidents est perçue comme une révolution aux États-Unis, dont le système d'éducation médicale est fondé sur l'immersion complète des futurs médecins. C'est la place des résidents dans le dispositif hospitalier qui est remise en question ainsi que la répartition des tâches au sein des équipes hospitalières [2-4]. Comme le conclut D.F. Weinstein: «de toutes façons, le terme de résident va bientôt devenir impropre » [4].

La réduction du temps de travail des internes/résidents est donc une tendance universelle, et la redéfinition de leur formation et de leurs missions une obligation. Ce qu'on entend par réduction est néanmoins bien différent aux États-Unis et en Europe. Au sein même de l'Europe, certains pays sont plus zélés que d'autres.

Quelle place pour la France, patrie des Droits de l'Homme, détentrice de «la meilleure médecine du monde » ailleurs qu'à la tête de ce juste combat? Dommage que la pénurie médicale vienne gâcher la fête. II faudra expliquer aux « usagers » des hôpitaux, bref, aux malades, d'aujourd'hui et de demain, que l'esclavage est enfin aboli. Nul doute qu'ils comprendront... $\diamond$

Working time of resident physicians

\section{RÉFÉRENCES}

1. Pickersgill T. The European working time directive for doctors in training. Br Med J 2001; 323: 1266.

2. Gaba DM, Howard SK. Patient safety: fatigue among clinicians and the safety of patients. $N$ Engl Med 2002; 347: 1249-55.

3. Drazen JM, Epstein AM. Rethinking medical training: the critical work ahead. N Engl J Med 2002; 347: 1271-2.

4. Weinstein DF. Duty hours for resident physicians: tough choices for teaching hospitals. N Engl J Med 2002; 347: 1275-8.

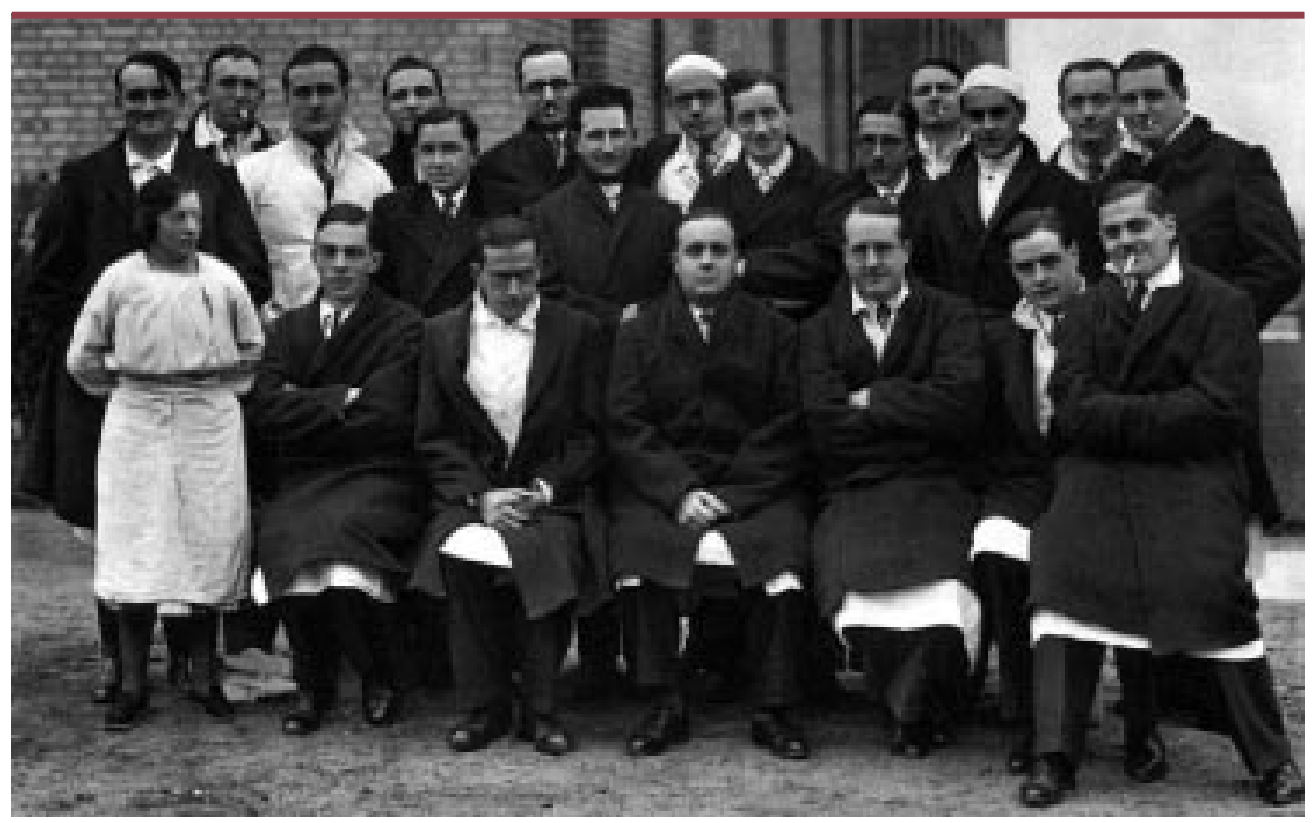

Internes de l'Hôpital Bichat (Paris, France), 1933. 